with the $\mathrm{T} \rightarrow \mathrm{C}$ transition in position 16,217 , this simply supports the view of the shared common origin of the ancestral settlers of Oceania and the New World. The absence of the other two Polynesian substitutions (mtDNA sites 16,247 and 16,261 ) in the New World points to a lack of significant contact between Polynesia and the Americas.

Our findings confirm the usefulness of molecular-genetic analysis of skeletal remains for addressing questions in prehistory and evolution. Although there are considerable technical problems in analysing ancient DNA, including DNA damage and artefacts caused by contamination, as well as the anecdotal nature of much of the data, these difficulties are not insuperable. The bones used in this study were stored for almost 40 years following their excavation by the Heyerdahl team and studied by several groups of physical anthropologists without special precautionš to avoid contamination. Nevertheless, unambiguous genetic information could be recovered from them.

\section{Erika Hagelberg}

Department of Biological Anthropology, University of Cambridge,

Cambridge CB2 3DZ, UK

Silvia Quevedo

Sección de Antropología,

Museo Nacional de Historia Natural,

Santiago de Chile, Chile

Daniel Turbon

Universitat de Barcelona, facultat de Biología,

Secció d'Antropología,

08028 Barcelona, Spain

\section{J. B. Clegg}

MRC Molecular Haematology Unit, Institute of Molecular Medicine,

John Radcliffe Hospital,

Oxford OX3 9DU, UK

\section{Hypothetical SisterKiller}

SIR - It was premature of Hurst in his News and Views article ${ }^{1}$ to accept Haig's claim $^{2}$ that a hypothetical meiotic drive element, SisterKiller, can lead to evolution from one-step to multi-step meiosis. The basis of Haig's claim is that a SisterKiller allele that causes a gamete to kill its sister gamete can invade and go to fixation in a population using one-step meiosis, whereas in a population using multi-step meiosis, SisterKiller cannot invade. Hurst concludes that SisterKiller could have caused the evolution of multi-step meiosis. The conditions under which this is true are more restrictive than Haig suggests.

Some simple algebraic models clarify the issues. Let $p$ be the frequency of SisterKiller in a haploid population using one-step meiosis to produce two gametes.
By hypothesis, a homozygote and a heterozygote for SisterKiller will produce only a single gamete, in both cases containing the SisterKiller allele. Assuming random mating, the recurrence equation for the frequency of the SisterKiller allele is

$$
p^{\prime}=\frac{p(2-p)}{2(1-p)+p^{2}}
$$

There are two equilibria, $p=1$ and $p=0$. The equilibrium at $p=0$ is unstable, indicating that SisterKiller can invade. Once present, SisterKiller will increase in frequency until fixed. However, when rare the change in frequency per generation is extremely low. Letting

$$
p=\frac{1}{N}
$$

where $N$ is the haploid population size, yields

$$
p^{\prime}=\frac{1}{N}+\frac{1}{2 N^{2}}+
$$

indicating that SisterKiller is nearly neutral when rare. Still, a SisterKiller allele may eventually drift up to the point where its advantage becomes significant and so can invade.

Now consider the same model as above but with non-random mating measured by the inbreeding coefficient $F$. The recurrence equation is readily modified ${ }^{3}$ to yield

$$
p^{\prime}=\frac{p(2-F-(1-F) p)}{2(1-p)+F p+(1-F) p^{2}}
$$

which has three equilibria, $p=1, p=0$ and

$$
p=\frac{F}{1-F}
$$

When the third, intermediate, equilibrium exists it is unstable and the equilibrium at $p=0$ becomes stable. Any amount of inbreeding $(F>0)$ brings in the intermediate equilibrium. SisterKiller when rare is then no longer nearly neutral but instead is deleterious. The high inbreeding that Hurst says would be required to prevent the invasion of SisterKiller is not necessary. SisterKiller, as defined by Haig, is unlikely to invade any large population using one-step meiosis.

It is possible to rescue SisterKiller by introducing a direct benefit for attacking (or consuming) the sister gamete. Haig

\section{Scientific Correspondence}

Scientific Correspondence is intended to provide a forum in which readers may raise points of a scientific character. Priority will be given to letters of fewer than 500 words and five references. suggests this variant but does not pursue it. Let $b$ be the direct benefit of SisterKiller's attack, such that the relative viability of SisterKiller gametes produced by SisterKiller homozygotes and heterozygotes is $1+b$. We then find that the resulting recurrence equation has three equilibria, $p=1, p=0$, and

$$
p=\frac{F+b(F-2)}{1-F-b(1-F)}
$$

When this third equilibrium is between 0 and 1 , the equilibrium at $p=0$ is rendered stable as above and SisterKiller cannot invade. The unstable intermediate equilibrium coalesces into the zero equilibrium which becomes unstable when

$$
b \geqslant \frac{F}{2-F}
$$

SisterKiller must derive benefit exceeding half the inbreeding coefficient to invade a population using one-step meiosis.

Finally, showing that a multi-step meiosis, when fixed, can prevent invasion of SisterKiller, does not support SisterKiller's role in the evolution of multi-step meiosis. Evaluating the impact of meioticdrive elements on the evolution of multistep meiosis from one-step meiosis via individual selection requires dynamic models describing the change in frequency of alleles controlling the method of meiosis, similar to those for the evolution of resistance of meiotic-drive elements through recombination ${ }^{4}$, modifiers ${ }^{5}$ and inter-deme selection ${ }^{6}$. These models have yet to be constructed and analysed, so SisterKiller's impact on the evolution of multi-step meiosis remains even more hypothetical than does SisterKiller itself.

\section{David L. Butcher}

Hong-Wen Deng

Department of Biology,

University of Oregon,

Eugene, Oregon 97403, USA

1. Hurst, L. Nature $365,206-207$ (1993)

2. Haig. D. J. theor. Biol. 163, 15-31(1993)

3. Wright, S. Evolution and the Genetics of Populations I Wright, S. Evolution and the Gen
(Univ. Chicago Press, 1969).

4. Haig, D. \& Grafen, A. J. theor. Biol. 153, 531-558 (1991)

5. Prout, T., Bundgaard, J. \& Bryant, S. Theor. Populat. Biol. 4, 446-465 (1973)

6. Leigh, E. G.Jr Proc. natn. Acad. Sci. U.S.A. 74, 45424546 (1977).

HAIG REPLIES - The argument that SisterKillers favour two-step meiosis was always intended to apply to the case of direct local benefits. Butcher and Deng's own results show that, in the absence of inbreeding, any local benefit whatsoever $(b>0)$ allows the invasion of a SisterKiller. Such a condition for successful sororicide does not seem prohibitive.

\section{David Haig}

Museum of Comparative Zoology,

Harvard University,

Cambridge, Massachusetts 02138, USA 